\title{
Second centralizers and autocommutator subgroups of automorphisms
}

\author{
M. Badrkhani Asl ${ }^{1}$ (D), Mohammad Reza R. Moghaddam*1,2,3 (D) \\ ${ }^{1}$ Department of Mathematics, Mashhad Branch, Islamic Azad University, Mashhad, Iran \\ ${ }^{2}$ Department of Mathematics, Khayyam University, Mashhad, Iran \\ ${ }^{3}$ Department of Pure Mathematics, Centre of Excellence in Analysis on Algebraic Structures (CEAAS), \\ Ferdowsi University of Mashhad, P.O. Box 1159, , Mashhad, 91775, Iran
}

\begin{abstract}
In 1994, Hegarty introduced the notion of $K(G)$ and $L(G)$, the autocommutator and autocentral subgroups of $G$, respectively. He proved that if $G / L(G)$ is finite, then so is $K(G)$ and for the converse he showed that the finiteness of $K(G)$ and $A u t(G)$ gives that $G / L(G)$ is also finite. In the present article, we construct a precise upper bound for the order of the autocentral factor group $G / L(G)$, when $K(G)$ is finite and $\operatorname{Aut}(G)$ is finitely generated. In 2012, Endimioni and Moravec showed that if the centralizer of an automorphism $\alpha$ of a polycyclic group $G$ is finite, then $L(G)$ and $G / K(G)$ are both finite. Finally, we show that if in a 2-auto-Engel polycyclic group $G$, there exist two automorphisms $\alpha_{1}$ and $\alpha_{2}$ such that $C_{G}\left(\alpha_{1}, \alpha_{2}\right)=\left\{g \in G \mid\left[g, \alpha_{1}, \alpha_{2}\right]=1\right\}$ is finite, then $L_{2}(G)$ and $G / K_{2}(G)$ are both finite.
\end{abstract}

Mathematics Subject Classification (2010). 20E36, 20F45, 20F28.

Keywords. polycyclic groups, auto-Engel group, autocentral and auocommutator subgroups.

\section{Introduction and preliminary results}

In 1904, I. Schur [11] showed that the finiteness of the central factor group of a given group implies that the derived subgroup is also finite. B. H. Neumann in 1951 [7] proved that the converse of Schur's result holds, for finitely generated groups. In 2005, K. Podoski and B. Szegedy [9] showed that for a given group $G$, if $\left[\gamma_{2}(G): \gamma_{2}(G) \cap Z(G)\right]=n$ then $\left[G: Z_{2}(G)\right] \leq n^{2 \log _{2} n}$. Note that the converse of Schur's theorem is not true in general. As a counterexample, consider the infinite extra special $p$-groups, for any odd prime number $p$. P. Niroomand [8] generalized Neumann's theorem as follows: If $\gamma_{2}(G)$ is finite and $G / Z(G)$ is finitely generated, then $[G: Z(G)] \leq\left|\gamma_{2}(G)\right|^{d(G / Z(G))}$, where $d(X)$ is the minimal number of generators of a group $X$. Also in 2010, B. Sury [12] proved that if the set of commutator elements $S$ of a group $G$ is finite and $G / Z(G)$ is finitely generated, then $[G: Z(G)] \leq|S|^{d(G / Z(G))}$. D. Gumber et.al [3] handled the case $G / Z(G)=\left\langle x_{1} Z(G), \ldots, x_{n} Z(G)\right\rangle$ such that $\left[x_{i}, G\right]$ is finite, for all $1 \leq i \leq n$ and showed that $[G: Z(G)] \leq \prod_{i=1}^{n}\left|\left[x_{i}, G\right]\right|$ and $\gamma_{2}(G)$ is finite.

\footnotetext{
*Corresponding Author.

Email addresses: m.r.moghaddam@khayyam.ac.ir, rezam@ferdowsi.um.ac.ir (M.R.R. Moghaddam)

Received: 19.12.2017; Accepted: 04.08.2018
} 
For an element $x \in G$ and automorphism $\alpha$ of $G,[x, \alpha]=x^{-1} x^{\alpha}$ is the autocommutator of $x$ and $\alpha$. So one may define

$$
K(G)=\langle[x, \alpha] \mid x \in G, \alpha \in \operatorname{Aut}(G)\rangle,
$$

and

$$
L(G)=\left\{x \in G \mid[x, \alpha]=x^{-1} \alpha(x)=1, \text { for all } \alpha \in A u t(G)\right\},
$$

which are called the autocommutator and autocentral subgroups of $G$, respectively. Clearly both subgroups are characteristic so that $K(G)$ contains the derived subgroup and $L(G)$ is contained in the centre of $G$ (see also $[4,6]$ for more information).

In [5], P. Hegarty proved that if $G / L(G)$ is finite, then so is $K(G)$. Also he showed that $G / L(G)$ is finite, whenever both $K(G)$ and $\operatorname{Aut}(G)$ are finite. In section 2, we construct an upper bound for the autocentral factor group of $G$, assuming its autocommutator subgroup is finite and the automorphism group of $G$ is finitely generated.

For any automorphism $\alpha$ of the group $G$, we define

$$
\begin{gathered}
C_{G}(\alpha)=\{x \in G \mid[x, \alpha]=1\}, \\
{[G, \alpha]=\langle[x, \alpha] \mid x \in G\rangle,}
\end{gathered}
$$

which are the centralizer of $\alpha$ in $G$ and the commutator subgroup of $G$ and $\alpha$. Clearly $[G, \alpha]$ is a subgroup of $[G, A u t(G)]=K(G)$ and $C_{G}(\alpha)$ contains the autocentre of $G$.

The $n^{\text {th }}$ autocommutator subgroup and $n^{\text {th }}$ autocentre of $G$ can be defined recursively, as follows:

$$
\begin{gathered}
K_{n}(G)=\left\langle\left[x, \alpha_{1}, \ldots, \alpha_{n}\right]=\left[\left[x, \alpha_{1}, \ldots, \alpha_{n-1}\right], \alpha_{n}\right] \mid x \in G, \alpha_{i} \in \operatorname{Aut}(G)\right\rangle, \\
L_{n}(G)=\left\{x \in G \mid\left[x, \alpha_{1}, \ldots, \alpha_{n}\right]=1, \quad \text { for all } \alpha_{i} \in \operatorname{Aut}(G)\right\} .
\end{gathered}
$$

So we obtain the following series

$$
\begin{gathered}
G=K_{0}(G) \supseteq K(G)=K_{1}(G) \supseteq K_{2}(G) \supseteq \cdots \supseteq K_{n}(G) \supseteq \cdots, \\
1=L_{0}(G) \subseteq L_{1}(G)=L(G) \subseteq \cdots \subseteq L_{n}(G) \subseteq \cdots .
\end{gathered}
$$

One observes that $K_{n}(G)$ and $L_{n}(G)$ are characteristic subgroups in $G$ such that $K_{n}(G)$ contains the $(n+1)^{s t}$ term of the lower central series $\gamma_{n+1}(G)$, and $L_{n}(G)$ is contained in the $n^{\text {th }}$ centre of $G, Z_{n}(G)$ (see [6] for more information).

\section{Upper bound for the autocentral factor groups}

In this section, we construct an upper bound for the autocentral factor group of a given group, when the autocommutator subgroup is finite and the automorphism group is finitely generated.

The following results of Hegarty [5] are needed for our further studies.

\section{Theorem 2.1.}

(a) [5, Theorem 1.1] If $[G: L(G)]$ is finite, then so are $K(G)$ and $\operatorname{Aut}(G)$.

(b) [5, Theorem 1.2] If $K(G)$ and Aut $(G)$ are both finite, then so is $[G: L(G)]$.

Note that Fournelle's example in [2] shows that the conclusion of part(b) is not true if the finiteness condition on $\operatorname{Aut}(G)$ is removed.

In the following, we extend this theorem of [5] to the case, where $\operatorname{Aut}(G)$ is finitely generated and construct a sharp upper bound for the order of $G / L(G)$, provided $K(G)$ is finite.

Theorem 2.2. Let the autocommutator subgroup $K(G)$ of a given group $G$ be finite of order $m$, and $d$ be the minimal number of generators of $A u t(G)$. Then

$$
|G / L(G)| \leq m^{d} .
$$


Proof. Let $\left\{\alpha_{1}, \ldots, \alpha_{d}\right\}$ be a minimal set of generators of automorphism group of a given group $G$. Define the following map

$$
f: G / L(G) \longrightarrow \underbrace{K(G) \times K(G) \times \cdots \times K(G)}_{d \text {-times }},
$$

given by $x L(G) \longmapsto\left(\left[x^{-1}, \alpha_{1}\right], \ldots,\left[x^{-1}, \alpha_{d}\right]\right)$, for all $x \in G$ and $\alpha_{1}, \ldots, \alpha_{d} \in A u t(G)$. The map $f$ is well-defined, for if $x_{1} L(G)=x_{2} L(G)$ for any $x_{1}, x_{2} \in G$, then $x_{1}^{-1} x_{2} \in L(G)$ and hence $\left[x_{1}^{-1} x_{2}, \alpha_{i}\right]=1$, for all $1 \leq i \leq d$. So it follows that $\left[x_{1}^{-1}, \alpha_{i}\right]=\left[x_{2}^{-1}, \alpha_{i}\right]$. To show the injectivity of $f$ assume $f\left(x_{1} L(G)\right)=f\left(x_{2} L(G)\right)$, which implies that $\left[x_{1}^{-1} x_{2}, \alpha_{i}\right]=1$, for all $1 \leq i \leq d$. Hence $x_{1}^{-1} x_{2} \in L(G)$, which shows that $f$ is injective. Therefore

$$
|G / L(G)| \leq m^{d} .
$$

The above map need not be onto. For example, the automorphism group of $D_{8}$ is isomorphic to itself. Hence $L\left(D_{8}\right)=\mathbb{Z}_{2}$ and $K\left(D_{8}\right)=\mathbb{Z}_{4}$, which imply that $\left|K\left(D_{8}\right)\right|^{d\left(D_{8}\right)}=$ 16 , while $\left|D_{8} / L\left(D_{8}\right)\right|=4$.

It should be noted that this bound is the best possible one, as the following example shows:

Let $\mathbb{Z}_{2}$ and $\mathbb{Z}_{4}$ be the cyclic groups of orders 2 and 4 , respectively. Then clearly $L\left(\mathbb{Z}_{2}\right)=$ $L\left(\mathbb{Z}_{4}\right)=\mathbb{Z}_{2}, K\left(\mathbb{Z}_{2}\right)=1$ and $K\left(\mathbb{Z}_{4}\right)=\mathbb{Z}_{2}$. Hence, in the both cases the bounds are attained.

\section{The second centralizers and autocommutator subgroups in auto-Engel groups}

An element $x$ of a group $G$ is called right $n$-auto-Engel if $[x, \underbrace{\alpha, \ldots, \alpha}_{n \text {-times }}]=1$, for all $\alpha \in$ $\operatorname{Aut}(G)$. The element $x$ is said to be left $n$-auto-Engel, whenever $[\alpha, \underbrace{x, \ldots, x}_{n-\text { times }}]=1$. A group $G$ is called $n$-auto-Engel if $[x, \underbrace{\alpha, \ldots, \alpha}_{n \text {-times }}]=[\alpha, \underbrace{x, \ldots, x}_{n-\text { times }}]=1$, for all $\alpha \in \operatorname{Aut}(G)$ and $x \in G$. (see also [10] for more details).

The next lemmas of [10] will be used frequently in our further investigations.

Lemma 3.1. [10, Lemma 3.2] Let $x$ be a right 2-auto-Engel element and $\alpha, \beta$ and $\gamma$ be arbitrary automorphisms of a given group $G$. Then

(1) $x$ is a left 2-auto-Engel element;

(2) $x^{\text {Aut }(G)}=\left\langle x^{\alpha}: \alpha \in A u t(G)\right\rangle$ is abelian and its elements are right (so left) 2-autoEngel elements;

(3) $[x, \alpha, \beta]=[x, \beta, \alpha]^{-1}$;

(4) $[x,[\alpha, \beta]]=[x, \alpha, \beta]^{2}$;

(5) $[x,[\alpha, \beta], \gamma]=1$.

Lemma 3.2. [10, Lemma 3.4] Let $G$ be a 2-auto-Engel group. Then the following properties hold, for all $x, y \in G, \alpha \in \operatorname{Aut}(G)$ and $n \in \mathbb{Z}$.

(1) $\left[x, x^{\alpha}\right]=1$;

(2) $\left[x, \alpha^{n}\right]=[x, \alpha]^{n}=\left[x^{n}, \alpha\right]$;

(3) $\left[x^{\alpha}, y\right]=\left[x, y^{\alpha}\right]$;

(4) $[\alpha, x, y]=[\alpha, y, x]^{-1}$.

Definition 3.3. For any automorphisms $\alpha_{1}$ and $\alpha_{2}$ of a group $G$, we call

$$
C_{G}\left(\alpha_{1}, \alpha_{2}\right)=\left\{x \in G \mid\left[x, \alpha_{1}, \alpha_{2}\right]=1\right\}
$$


the second centralizer of $\alpha_{1}$ and $\alpha_{2}$. Clearly, if $G$ is abelian, then $C_{G}\left(\alpha_{1}, \alpha_{2}\right)$ is a subgroup of $G$.

Lemma 3.4. Let $G$ be a 2-auto-Engel group, $\alpha_{1}$ and $\alpha_{2}$ be arbitrary automorphisms of $G$. Then $C_{G}\left(\alpha_{1}, \alpha_{2}\right)$ and $\left[G, \alpha_{1}, \alpha_{2}\right]=\left\langle\left[x, \alpha_{1}, \alpha_{2}\right] \mid x \in G\right\rangle$ are both normal subgroups of $G$.

Proof. For any elements $x_{1}, x_{2} \in C_{G}\left(\alpha_{1}, \alpha_{2}\right)$, then by Lemma 3.1(3) and Lemma 3.2(2), we have

$$
\begin{aligned}
{\left[x_{1} x_{2}^{-1}, \alpha_{1}, \alpha_{2}\right]=} & {\left[\left[x_{1}, \alpha_{1}\right]^{x_{2}^{-1}}\left[x_{2}^{-1}, \alpha_{1}\right], \alpha_{2}\right] } \\
& =\left[\left[x_{1}, \alpha_{1}\right]\left[x_{1}, \alpha_{1}, x_{2}^{-1}\right]\left[x_{2}^{-1}, \alpha_{1}\right], \alpha_{2}\right] \\
& =\left[\left[x_{1}, \alpha_{1}, x_{2}^{-1}\right]\left[x_{2}^{-1}, \alpha_{1}\right], \alpha_{2}\right] \\
& =\left[x_{1}, \alpha_{1}, x_{2}^{-1}, \alpha_{2}\right]^{\left[x_{2}^{-1}, \alpha_{1}\right]} \\
& =\left[x_{1}, \alpha_{1}, \phi_{x_{2}^{-1}}, \alpha_{2}\right]^{\left[x_{2}^{-1}, \alpha_{1}\right]} \\
& =\left(\left[x_{1}, \alpha_{1}, \alpha_{2}, \phi_{x_{2}^{-1}}\right]^{-1}\right)^{\left[x_{2}^{-1}, \alpha_{1}\right]}=1,
\end{aligned}
$$

where $\phi_{x_{2}^{-1}}$ denotes the inner automorphism induced by $x_{2}^{-1}$. Therefore, $x_{1} x_{2}^{-1} \in$ $C_{G}\left(\alpha_{1}, \alpha_{2}\right)$ and hence it is a subgroup.

Now, to prove the normality of $C_{G}\left(\alpha_{1}, \alpha_{2}\right)$, take any element $x \in C_{G}\left(\alpha_{1}, \alpha_{2}\right)$ and $g \in G$, then by Lemma 3.1(3) we have

$$
\begin{aligned}
{\left[x^{g}, \alpha_{1}, \alpha_{2}\right] } & =\left[x[x, g], \alpha_{1}, \alpha_{2}\right] \\
& =\left[\left[x, \alpha_{1}\right]^{[x, g]}\left[x, g, \alpha_{1}\right], \alpha_{2}\right] \\
& =\left[\left[x, \alpha_{1}\right]\left[x, \alpha_{1},[x, g]\right]\left[x, g, \alpha_{1}\right], \alpha_{2}\right] \\
& =\left[\left[x, \alpha_{1},[x, g]\right]\left[x, g, \alpha_{1}\right], \alpha_{2}\right] \\
& =\left[\left[x, \alpha_{1},[x, g]\right], \alpha_{2}\right]^{\left[x, g, \alpha_{1}\right]}\left[x, g, \alpha_{1}, \alpha_{2}\right] \\
& =\left[x, \alpha_{1}, \phi_{[x, g]}, \alpha_{2}\right]^{\left[x, g, \alpha_{1}\right]}\left[x, \phi_{g}, \alpha_{1}, \alpha_{2}\right] \\
& =\left(\left[x, \alpha_{1}, \alpha_{2}, \phi_{[x, g]}\right]^{-1}\right)^{\left[x, g, \alpha_{1}\right]}\left[x, \alpha_{1}, \alpha_{2}, \phi_{g}\right]=1,
\end{aligned}
$$

and hence $C_{G}\left(\alpha_{1}, \alpha_{2}\right)$ is normal in $G$.

For all $\left[x, \alpha_{1}, \alpha_{2}\right] \in\left[G, \alpha_{1}, \alpha_{2}\right]$ and $g \in G$, we have

$$
\left[x, \alpha_{1}, \alpha_{2}\right]^{g}=\left[x, \alpha_{1}, \alpha_{2}\right]\left[x, \alpha_{1}, \alpha_{2}, g\right] .
$$

Now, it is enough to show that $\left[x, \alpha_{1}, \alpha_{2}, g\right] \in\left[G, \alpha_{1}, \alpha_{2}\right]$. Easily by Lemmas 3.1(3) and $3.2(2)$, we write

$$
\begin{aligned}
{\left[x, \alpha_{1}, \alpha_{2}, g\right]=\left[x, \alpha_{1}, \alpha_{2}, \phi_{g}\right] } & =\left[x, \alpha_{1}, \phi_{g}, \alpha_{2}\right]^{-1} \\
& =\left[\left[x, \phi_{g}, \alpha_{1}\right]^{-1}, \alpha_{2}\right]^{-1} \\
& =\left[x, g, \alpha_{1}, \alpha_{2}\right] \in\left[G, \alpha_{1}, \alpha_{2}\right] .
\end{aligned}
$$

The following lemma is useful for our further studies.

Lemma 3.5. (1) If $\alpha_{1}$ and $\alpha_{2}$ are any automorphisms of a 2-auto-Engel group $G$, then $C_{G}\left(\alpha_{i}\right) \leq C_{G}\left(\alpha_{1}, \alpha_{2}\right)$ for $i=1,2$ and $\left[G, \alpha_{1}, \alpha_{2}\right] \leq\left[G, \alpha_{i}\right]$.

(2) If $N$ is $\alpha_{1}$ and $\alpha_{2}$-invariant subgroup of a finite abelian group $G$, then $\left|C_{G / N}\left(\overline{\alpha_{1}}, \overline{\alpha_{2}}\right)\right| \leq\left|C_{G}\left(\alpha_{1}, \alpha_{2}\right)\right|$, where $\overline{\alpha_{1}}$ and $\overline{\alpha_{2}}$ are induced automorphisms by $\alpha_{1}$ and $\alpha_{1}$, respectively. 
Proof. Part (1) follows easily by the above discussion.

(2) By Definition 3.3,

$$
\begin{aligned}
C_{G / N}\left(\overline{\alpha_{1}}, \overline{\alpha_{2}}\right) & =\left\{x N \in G / N \mid\left[x N, \overline{\alpha_{1}}, \overline{\alpha_{2}}\right]=N\right\} \\
& =\left\{x N \in G / N \mid\left[x, \alpha_{1}, \alpha_{2}\right] \in N\right\} .
\end{aligned}
$$

Now, we define the map $\theta: G \longrightarrow N$ given by $x \longmapsto\left[x, \alpha_{1}, \alpha_{2}\right]$, for all $x \in G$. Clearly $\theta$ is a homomorphism and hence $G / \operatorname{ker} \theta \cong \theta(G) \leq N$. Therefore $\left|C_{G / N}\left(\overline{\alpha_{1}}, \overline{\alpha_{2}}\right)\right| \leq|G / N| \leq$ $|\operatorname{ker} \theta|=\left|C_{G}\left(\alpha_{1}, \alpha_{2}\right)\right|$.

Corollary 3.6. Let $\alpha_{1}$ and $\alpha_{2}$ be automorphisms of a 2-auto-Engel polycyclic group $G$ such that $\alpha_{1}$ is of order 2 and $C_{G}\left(\alpha_{1}, \alpha_{2}\right)$ is finite, then the derived autocommutator subgroup $\left[G, \alpha_{1}, \alpha_{2}\right]^{\prime}$ is also finite.

Proof. The proof follows by using Lemma 3.3 and [1, Theorem 1].

Corollary 3.7. Let $\alpha_{1}$ and $\alpha_{2}$ be two automorphisms of a 2-auto-Engel soluble group $G$ such that $\alpha_{1}$ is a fixed point free automorphism of order 2 , then $\left[G, \alpha_{1}, \alpha_{2}\right]$ is abelian.

Proof. As $G$ is soluble, Theorem 6 of [1] says that; for any fixed point free automorphism $\alpha_{1}$ of order 2, the subgroup $\left[G, \alpha_{1}\right]$ is abelian. Now, by Lemma 3.5(1), $\left[G, \alpha_{1}, \alpha_{2}\right]$ must be abelian.

G. Endimioni and P. Moravec in [1], proved the following result.

Theorem 3.8. [1, Theorem 2] Let $\alpha$ be an automorphism of a polycyclic group G. If $C_{G}(\alpha)$ is finite, then so is $G /[G, \alpha]$.

In our terminology, we have the following corollary of the above theorem.

Corollary 3.9. Let $\alpha$ be an automorphism of a polycyclic group $G$. If $C_{G}(\alpha)$ is finite, then so are $L(G)$ and $G / K(G)$.

If we assume additionally in Theorem 3.8 that the group $G$ is 2-auto-Engel, then we are able to show that the finiteness of $C_{G}\left(\alpha_{1}, \alpha_{2}\right)$ implies that $G /\left[G, \alpha_{1}, \alpha_{2}\right]$ is also finite (see Theorem 3.12 below). It is equivalent to saying that, if $C_{G}\left(\alpha_{1}, \alpha_{2}\right)$ is finite, then so are $L_{2}(G)$ and $G / K_{2}(G)$.

Remark 3.10. We shall make frequently use of the following well-known fact, without further references: if $H$ is a finitely generated characteristic subgroup of a given group $A$ and $H_{1}$ is a normal subgroup of $H$ of finite index, then $H_{1}$ contains a characteristic subgroup $\mathrm{H}_{2}$ of $A$ of finite index in $H$.

The following technical lemmas are needed in proving our main result.

Lemma 3.11. Let $A$ be a finitely generated abelian group and $\alpha_{1}$ and $\alpha_{2}$ be any automorphisms of $A$, with $C_{A}\left(\alpha_{1}, \alpha_{2}\right)$ is finite. Then $A$ contains a characteristic subgroup of finite index, such that its elements are of the form $\left[a, \alpha_{1}, \alpha_{2}\right]$, for some $a \in A$.

Proof. Define the map $\theta: A \longrightarrow A$ given by $a \longmapsto\left[a, \alpha_{1}, \alpha_{2}\right]$, for all $a \in A$. The map $\theta$ is a homomorphism, as $A$ is abelian. Clearly, the kernel of $\theta$ is $C_{A}\left(\alpha_{1}, \alpha_{2}\right)$, which is finite by the assumption. Then $A$ and $\theta(A)$ have the same torsion-free rank and hence the index of $\theta(A)$ in $A$ is finite. Clearly, the elements of $\theta(A)$ are of the required form. Now, by the above Remark and taking $H=A$, as $A$ is finitely generated and $\theta(A)$ is a normal subgroup of finite index in $A$, then $\theta(A)$ contains a characteristic subgroup of finite index in $A$ such that its elements are also of the form $\left[a, \alpha_{1}, \alpha_{2}\right]$, for some $a \in A$. 
Lemma 3.12. Let $G$ be an abelian group and $K$ be a finitely generated characteristic subgroup of $G$. Let $\alpha_{1}$ and $\alpha_{2}$ be two automorphisms of $G$ such that $C_{G}\left(\alpha_{1}, \alpha_{2}\right)$ is finite. Then $C_{G / K}\left(\overline{\alpha_{1}}, \overline{\alpha_{2}}\right)$ is also finite, where $\overline{\alpha_{1}}$ and $\overline{\alpha_{2}}$ are automorphisms of $G / K$ induced by the automorphisms $\alpha_{1}$ and $\alpha_{2}$, respectively.

Proof. The result is trivially true, when the group $G$ is finite. Otherwise, we assume that $K$ is finite and put $C_{G / K}\left(\overline{\alpha_{1}}, \overline{\alpha_{2}}\right)=C / K$, then we define the map $\theta: C \longrightarrow K$ given by $c \longmapsto\left[c, \alpha_{1}, \alpha_{2}\right]$, for all $c \in C$. Clearly $\theta\left(c_{1}\right)=\theta\left(c_{2}\right)$ if and only if $c_{1} c_{2}^{-1} \in C_{G}\left(\alpha_{1}, \alpha_{2}\right)$, which implies that $C$ is finite. Hence $|C| \leq|K| \times\left|C_{G}\left(\alpha_{1}, \alpha_{2}\right)\right|$. Therefore $\left|C_{G / K}\left(\overline{\alpha_{1}}, \overline{\alpha_{2}}\right)\right|=$ $[C: K] \leq\left|C_{G}\left(\alpha_{1}, \alpha_{2}\right)\right|<\infty$, and hence the claim. Now, we assume that $K$ is finitely generated. Since $\left|C_{K}\left(\alpha_{1}, \alpha_{2}\right)\right| \leq\left|C_{G}\left(\alpha_{1}, \alpha_{2}\right)\right|<\infty$, Lemma 3.11 gives that $K$ contains a characteristic subgroup $K_{0}$ of finite index such that for all $k_{0} \in K_{0}$, there is a unique $y \in K$ where $k_{0}=\left[y, \alpha_{1}, \alpha_{2}\right]$. Let $\alpha_{1}{ }^{\prime}$ and $\alpha_{2}{ }^{\prime}$ be the automorphisms of $G / K_{0}$ induced by $\alpha_{1}$ and $\alpha_{2}$, respectively. We show that $C_{G / K_{0}}\left(\alpha_{1}{ }^{\prime}, \alpha_{2}{ }^{\prime}\right)$ is finite. To prove this, take an element $x K_{0} \in C_{G / K_{0}}\left(\alpha_{1}{ }^{\prime}, \alpha_{2}{ }^{\prime}\right)$, then we have $\left[x, \alpha_{1}, \alpha_{2}\right] \in K_{0}$. Assume $\left[x, \alpha_{1}, \alpha_{2}\right]=\left[y, \alpha_{1}, \alpha_{2}\right]$, for some $y \in K$. It follows that $\left[x y^{-1}, \alpha_{1}, \alpha_{2}\right]=1$. Now, since the index of $K_{0}$ in $K$ is finite, we may assume that $K=t_{1} K_{0} \cup \cdots \cup t_{n} K_{0}$. So $y=t_{i} k^{\prime}$, for some $k^{\prime} \in K_{0}$ and hence $\left[x\left(t_{i} k^{\prime}\right)^{-1}, \alpha_{1}, \alpha_{2}\right]=1$. Therefore $x\left(t_{i} k^{\prime}\right)^{-1} \in C_{G}\left(\alpha_{1}, \alpha_{2}\right)$ and so $x K_{0}=u t_{i} K_{0}$, for some $u \in C_{G}\left(\alpha_{1}, \alpha_{2}\right)$. Therefore the group $C_{G / K_{0}}\left(\alpha_{1}{ }^{\prime}, \alpha_{2}{ }^{\prime}\right)$ is finite. Now, assume $\overline{\alpha_{1}}$ and $\overline{\alpha_{2}}$ are induced by the automorphisms $\alpha_{1}^{\prime}$ and $\alpha_{2}^{\prime}$ on $G / K$. As $K / K_{0}$ is finite, we conclude that the group $C_{G / K}\left(\overline{\alpha_{1}}, \overline{\alpha_{2}}\right)$ is finite by the first part of the lemma.

Note that in the preceding lemma, in the case where $K$ is finitely generated, one can not hope the inequality $\left|C_{G / K}\left(\overline{\alpha_{1}}, \overline{\alpha_{2}}\right)\right| \leq\left|C_{G}\left(\alpha_{1}, \alpha_{2}\right)\right|$, if we take $G=\mathbb{Z}, K=2 \mathbb{Z}$ and $\alpha_{1}=\alpha_{2}=\alpha: x \longmapsto-x$, then we have $\left|C_{G}(\alpha, \alpha)\right|=1$, while $\left|C_{G / K}(\bar{\alpha}, \bar{\alpha})\right|=2$.

Now, we are able to prove the following result.

Theorem 3.13. Let $G$ be a polycyclic 2-auto-Engel group and $\alpha_{1}, \alpha_{2}$ be two automorphisms of $G$. If $C_{G}\left(\alpha_{1}, \alpha_{2}\right)$ is finite, then so is $G /\left[G, \alpha_{1}, \alpha_{2}\right]$.

Proof. We proceed by induction on the derived length $d$ of $\left[G, \alpha_{1}, \alpha_{2}\right]$. If $d=0$, then $\left[G, \alpha_{1}, \alpha_{2}\right]=1$. This implies that $C_{G}\left(\alpha_{1}, \alpha_{2}\right)=G$ and hence $G$ is finite, which gives the result. Put $K=\left[G, \alpha_{1}, \alpha_{2}\right]^{(d-1)}$ and it can be checked that $K$ is finitely generated abelian. Now we can apply Lemma 3.12 and get the finiteness of $C_{G / K}\left(\overline{\alpha_{1}}, \overline{\alpha_{2}}\right)$, where $\overline{\alpha_{1}}$ and $\overline{\alpha_{2}}$ are induced automorphisms by $\alpha_{1}$ and $\alpha_{2}$. Using induction hypothesis, we have $[G / K$ : $\left.\left[G / K, \overline{\alpha_{1}}, \overline{\alpha_{2}}\right]\right]$ is finite, which implies the finiteness of $G /\left[G, \alpha_{1}, \alpha_{2}\right]$, as required.

The above theorem gives our main result of this section.

Corollary 3.14. Let $G$ be a polycyclic 2-auto-Engel group with two automorphisms $\alpha_{1}$ and $\alpha_{2}$ such that $C_{G}\left(\alpha_{1}, \alpha_{2}\right)$ is finite. Then $L_{2}(G)$ and $G / K_{2}(G)$ are both finite.

The following technical lemma is needed to prove our final result.

Lemma 3.15. Let $x$ and $y$ be arbitrary elements of a group $G$. Then for any automorphism $\alpha$ of $G,\left[y,\left[\phi_{x}, \alpha\right]\right]=[y,[x, \alpha]]$.

Proof.

$$
\begin{aligned}
{\left[y,\left[\phi_{x}, \alpha\right]\right] } & =y^{-1} y^{\left[\phi_{x}, \alpha\right]}=y^{-1} y^{\left(\phi_{x}^{-1} \alpha^{-1} \phi_{x} \alpha\right)} \\
& =y^{-1}\left(y^{x^{-1}}\right)^{\alpha^{-1} \phi_{x} \alpha}=y^{-1}\left(\left(y^{\alpha^{-1}}\right)^{\left(x^{-1}\right)^{\alpha^{-1}}}\right)^{\phi_{x} \alpha} \\
& =y^{-1}\left(\left(y^{\alpha^{-1}}\right)^{\left(x^{-1}\right)^{-1}} x\right)^{\alpha}=y^{-1} y^{x^{-1} x^{\alpha}}=y^{-1} y^{[x, \alpha]} \\
& =[y,[x, \alpha]]
\end{aligned}
$$


Corollary 3.16. Let $G$ be a 2-auto-Engel polycyclic group and $C_{G}\left(\alpha_{1}, \alpha_{2}\right)$ is finite, for any automorphisms $\alpha_{1}$ and $\alpha_{2}$ of $G$. Then $G / Z(G)$ is finite.

Proof. Let $x, y \in G, \alpha, \beta \in \operatorname{Aut}(G)$ and $\phi_{x}$ be the inner automorphism induced by $x$. As $G$ is 2-auto-Engel group, by Lemma 3.1(5), $\left[y,\left[\phi_{x}, \alpha\right], \beta\right]=1$ and Lemma 3.14 gives $[y,[x, \alpha], \beta]=1$. Now using Lemma $3.1(3)[x, \alpha, y, \beta]=1$ and so $[x, \alpha, \beta, y]=1$. This shows that $K_{2}(G)$ is contained in the centre of $G$. Hence the result is obtained by corollary 3.13 .

Open problem: Let $G$ be an n-auto-Engel polycyclic group with $C_{G}\left(\alpha_{1}, \alpha_{2}, \ldots, \alpha_{n}\right)$ is finite, for any automorphisms $\alpha_{1}, \alpha_{2}, \ldots, \alpha_{n}$ of the group $G$. Are $L_{n}(G)$ and $G / K_{n}(G)$ finite?

Acknowledgment. The authors would like to thank the referee for careful reading and constructive suggestions, which improve the article.

\section{References}

[1] G. Endimioni and P. Moravec, On the centralizer and the commutator subgroup of an automorphism, Monatshefte für Mathematik, 167, 165-174, 2012.

[2] T.A. Fournelle, Elementary abelian p-groups as automorphisms groups of infinite groups II, Houston J. Math. 9, 269-276, 1983.

[3] D. Gumber, H. Kalra and S. Single, Automorphisms of groups and converse of Schur's theorem, at: http://arXiv.org/math/arXiv:1303.4966v1.

[4] P.V. Hegarty, The absolute centre of a group, J. Algebra, 169, 929-935, 1994.

[5] P.V. Hegarty, Autocommutator subgroups of finite groups, J. Algebra, 190, 556-562, 1997.

[6] M.R.R. Moghaddam, F. Parvaneh and M. Naghshineh, The lower autocentral series of abelian groups, Bull. Korean Math. Soc. 48, 79-83, 2011.

[7] B.H. Neumann, Groups with finite classes of conjugate elements, Proc. London Math. Soc. 3 (1), 178-187, 1951.

[8] P. Niroomand, The converse of Schur's theorem, Arch. Math. 94, 401-403, 2010.

[9] K. Podoski and B. Szegedy, Bounds for the index of the centre in capable groups, Proc. Amer. Math. Soc. 133, 3441-3445, 2005.

[10] H. Safa, M. Farrokhi D.G. and M.R.R. Moghaddam, Some properties of 2-auto-Engel groups, Houston J. Math. 44 (1), 31-48, 2018.

[11] I. Schur, Uber die darstellung der endlichen grouppen durch gebrochene lineare substiutionen, J. Reine Angew. Math. 127, 20-50, 1904.

[12] B. Sury, A generalization of a converse to Schur's theorem, Arch. Math. 95, 317-318, 2010 . 